\title{
First results of total attenuation distributions and fade dynamics from the Alphasat propagation experiment in Madrid
}

\author{
D. Pimienta-del-Valle ${ }^{(1)}$, A. Benarroch* ${ }^{(1)}$, J.M. Riera ${ }^{(1)}$, P. Garcia-del-Pino ${ }^{(2)}$ \\ (1) ETSI de Telecomunicación, Universidad Politécnica de Madrid (UPM), Spain \\ (2) ETS de Ingeniería y Sistemas de Telecomunicación, Universidad Politécnica de Madrid (UPM), Spain
}

\begin{abstract}
Experimental results in satellite propagation on millimeter-wave band have an important role in the development of propagation channel models for these frequencies. Two full years of measurements of the Qband beacon from the Alphasat satellite are available in Madrid, Spain, as part of a propagation study carried out by Universidad Politécnica de Madrid. In this paper, the main objective is to present the first results of these two years, specifically total attenuation distributions and fade dynamics. Results of fade dynamics are compared with prediction models. Experiment characteristics and data processing details are also presented. When compared with the results of other long-term experiments, it can be concluded that more measurements are necessary in order to deliver more reliable results.
\end{abstract}

\section{Introduction}

Due to the future saturation of the SHF spectrum (3-30 $\mathrm{GHz}$ ) and the increasing demand in data rate in the last years, academia, industry, and global standardization bodies have taken a growing interest in millimeter waves [1]. At these frequencies, various atmospheric factors may produce significant signal degradation or fades. This is why one important line of research is the development of propagation channel models. Satellite communication is one of the main applications of millimeter-wave bands. To produce satellite-propagation channel models a good understanding of the slant path propagation at these frequencies is necessary. In order to improve such understanding, propagation experiments are carried out. In these experiments several statistical parameters are relevant, like total and excess attenuation or fade durations, among others.

As part of a propagation study carried out throughout Europe by the ASAPE (Alphasat Aldo Paraboni Propagation Experimenters) members, the GTICRadiocommunications Group of Universidad Politécnica de Madrid (UPM) is performing measurements of the signal received from the Alphasat satellite beacon in the Q-band (40 GHz) since April 2014. At this moment, two complete years are available: year 1 from April 1st, 2014 to March 31, 2015 and year 2 from April 1st, 2015 to March 31, 2016. The third year will be completed by the time of the General Assembly. The measurements of the first two years have been validated and processed, and some results have been obtained, like excess and total attenuation time series [2] and fade dynamics statistics.

In this paper the main results of the first (attenuation) and second (fade dynamics) order statistical analysis obtained from the two years of data measurements are presented. The document is organized as follows: the main parameters of the experiment are described in Section 2, some data processing characteristics are provided in Section 3. Results on total attenuation are presented in Section 4. Second order statistics are provided and discussed in Section 5. Some conclusions are drawn in Section 6.

\section{Experiment characteristics}

The beacon receiver installed on the rooftop of a UPM building takes the transmitting signal in Q-band from the payload of the Alphasat satellite. The main characteristics of the experiment carried out are summarized in Table I. An extended version can be found in [3].

Table I. Main technical data of the Q-band propagation experiment.

\begin{tabular}{|l|c|}
\hline \multicolumn{1}{|c|}{ Technical Parameter } & Value \\
\hline Satellite position & $25^{\circ} \mathrm{E}$ \\
\hline Beacon frequency & Linear, $45^{\circ}$ tilted \\
\hline Polarization & $40.5^{\circ} \mathrm{N}, 3.7^{\circ} \mathrm{W}, 630 \mathrm{~m}$ amsl \\
\hline Site coordinates and height & $0.935 \mathrm{~m}$ and $48.3 \mathrm{~dB}$ \\
\hline Link azimuth and elevation & $21.7 \mathrm{~dB} / \mathrm{K}$ \\
\hline Antenna diameter and gain & $18.78 \mathrm{~Hz}$ \\
\hline G/T in deep fade conditions & $22 \mathrm{dBHz}(\mathrm{estimated})$ \\
\hline Measurement sampling rate & $37.8 \mathrm{~dB}$ \\
\hline Receiver threshold & $99.994 \%$ \\
\hline Rain margin & $\begin{array}{l}\text { Estimated availability of the } \\
\text { experiment against rain fades } \\
\text { (average year) }\end{array}$
\end{tabular}

\section{Data processing}

Raw data of the received power obtained from the beacon with a sampling frequency of 18.78 samples/s are preprocessed to produce files with the same amount of data 
samples per day. To do so, daily raw data are synchronized and combined with a normalized time base that allows the obtaining of such amount. Also, the new samples are tagged with one of the possible values of a flag vector: Valid data, Invalid data, Doubtful data (Receiver margin exceeded), Lack of measurements, Interpolated data and Corrected data. These values of the flag can be used in later phases of the processing.

To obtain excess attenuation, a reference level must be calculated. This reference corresponds to the clear-sky average signal and is obtained in two different ways. For events (rain and clouds along the propagation path) it is calculated on the basis of the signal level recorded before and after the event, as well as in some intermediate points when clear-sky conditions are met. The selection of these points is made manually by an experienced operator. For the rest of the time, the reference is generated by low-pass filtering the signal and extracting the slow variation due to periodical effects.

The time series of excess attenuation are obtained later by the subtraction of the pre-processed measurement to the reference level. An example of a time series of excess attenuation for a complete day is shown in Figure 1. This includes clear-air, clouds, rain and scintillation effects. The reference level corresponds to clear-air conditions and includes components of gas attenuation.

Excess attenuation must be combined with gas attenuation in order to obtain the time series of total attenuation. To do so, gas attenuation is derived from ZTD (Zenith Total Delay) data. This information is obtained from the GNSS receiver at the ESA (European Space Agency) station of Villafranca del Castillo, separated only by $16 \mathrm{~km}$ from the UPM site and at a similar altitude. ZTD data with a sampling interval of 5 minutes are freely available through the IGS network and can be used to calculate the water vapor content of the atmosphere, and then to estimate gas attenuation. The procedure is described in [2]. The time series of total attenuation for the day taken as an example is also shown in Figure 1. The difference between the two time series corresponds to gas attenuation, which has a slow variation throughout the day.

\section{Attenuation statistics}

There are three important time-series sets from which statistical results that can be calculated: total attenuation excess attenuation and excess attenuation using only the identified events of cloud or rain attenuation.

For the two years under study, the receiver data availability has being very high, as is shown in Table II. This parameter is obtained as the ratio between the time of valid measurements and the total time of the year. The total attenuation data availability is lower than others due to the lack of GNSS data in a few consecutive days in Year 1 .
Table II. Data availability for the different statistics.

\begin{tabular}{|c|c|c|}
\hline Data & Year 1 & Year 2 \\
\hline $\begin{array}{c}\text { Excess attenuation } \\
\text { Excess attenuation during } \\
\text { events }\end{array}$ & $98.0 \%$ & $96.1 \%$ \\
\hline Total attenuation & $96.4 \%$ & $97.7 \%$ \\
\hline
\end{tabular}

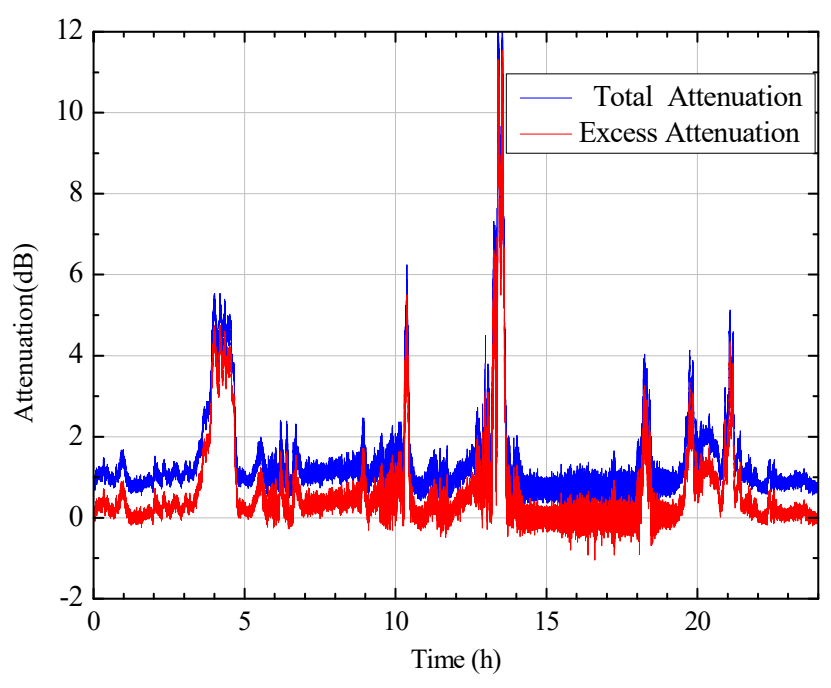

Figure 1. Time series of excess and total attenuation, for June $15^{\text {th }}, 2015$.

Figures 2 and 3 show the complementary cumulative distribution functions (CCDFs) of total attenuation for all months of the two years of measurements. In both years the margin was exceeded in October, and for the second year June and August also report outage periods.

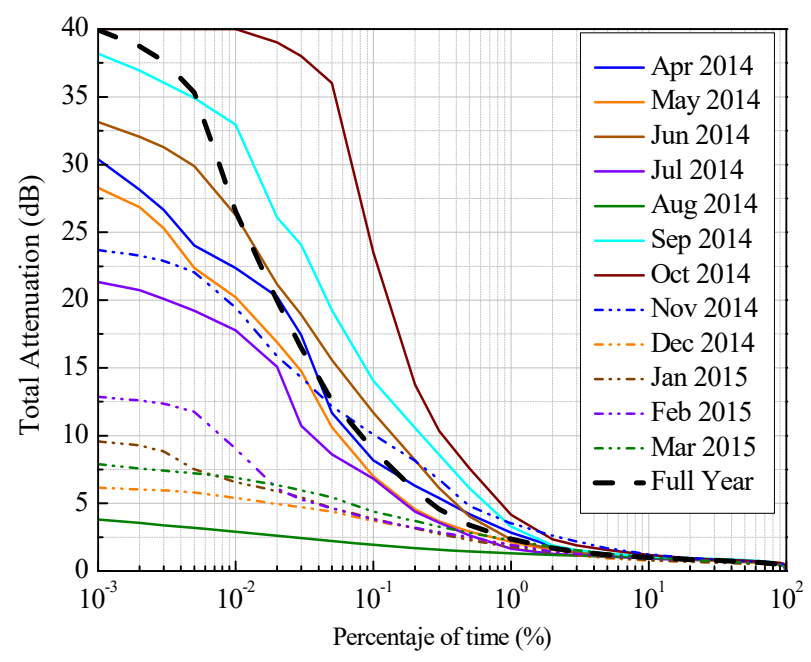

Figure 2. Total attenuation distribution in year 1.

A visual inspection of Figures 2 and 3 can conclude that a high year-to-year variability exists, which is a characteristic of the climatic conditions in Madrid 
reported in [4], reinforcing the need of high-duration experiments. These distributions show a shifting upwards by a few tenths of $\mathrm{dB}$ in comparison with the distributions of excess attenuation (not shown, available in [2]), an amount that corresponds to gas attenuation, which is present at all time percentages.

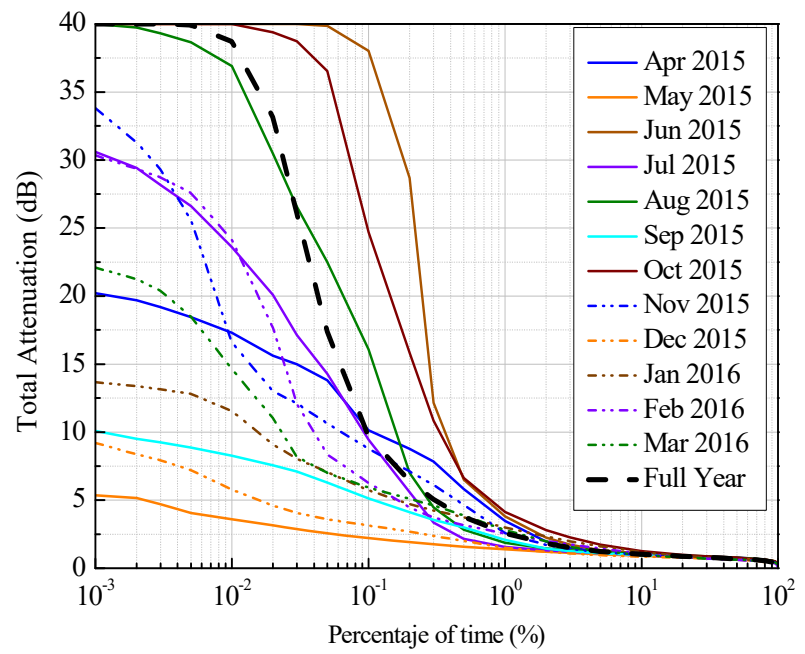

Figure 3. Total attenuation distribution in year 2 .

Differences are observed between the percentages of outages caused by the margin exceedance in each year, $0.003 \%$ and $0.01 \%$ for year 1 and 2 , respectively. These values are similar to the value of $0.006 \%$ obtained from propagation models and the technical data shown in Table I.

\section{Fade duration results}

Channel dynamics is a key element in the design of a control loop of propagation impairment mitigation techniques (PIMT). One of the parameters that allows the characterization of channel dynamics is fade durations, (as the time between two consecutive crosses of a threshold value of the received signal level).

To characterize the fade dynamics, Rec. ITU-R P. 1623 [5] provides the following conditioned distribution functions:

1. $P(d>D \mid a>A)$, that is, the probability of occurrence of fades of duration $d$ longer than $D(\mathrm{~s})$, given that the attenuation $a$ is greater than $A(\mathrm{~dB})$.

2.F $(d>D \mid a>A)$, that is, the cumulative exceedance probability, or, equivalently, the total fraction (between 0 and $100 \%$ ) of fade time due to fades of duration $d$ longer than $D(\mathrm{~s})$, given that the attenuation $a$ is greater than $A$ (dB).

This Recommendation proposes a method to calculate the fade duration statistics from total attenuation time series. Also, the Communications Research Centre Canada
(CRC) proposed in [6] a method to obtain $P(d>D \mid a>$ A).

In Figure 4 the distribution of the average-year number of fade event is shown (Exp), which is compared with the values obtained with the ITU method. The ITU values are obtained with the Recommendation method [5] using the experimental attenuation distribution for the same period. Particularly good approximations are obtained with the 5and $10-\mathrm{dB}$ curves, although the ITU model predicts most of the time smaller number of fades than those observed in the experiment.

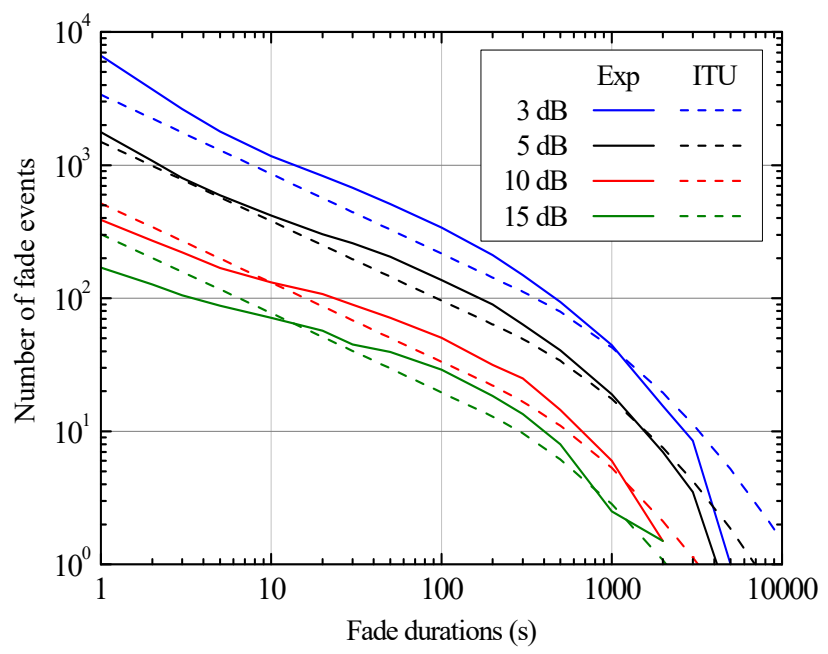

Figure 4. Number of events exceeding a given duration (average year).

Figure 5 shows the comparison of the conditional distributions $P(d>D \mid a>A)$ obtained from the experimental data and the two prediction methods (ITU and $\mathrm{CRC}$ ).

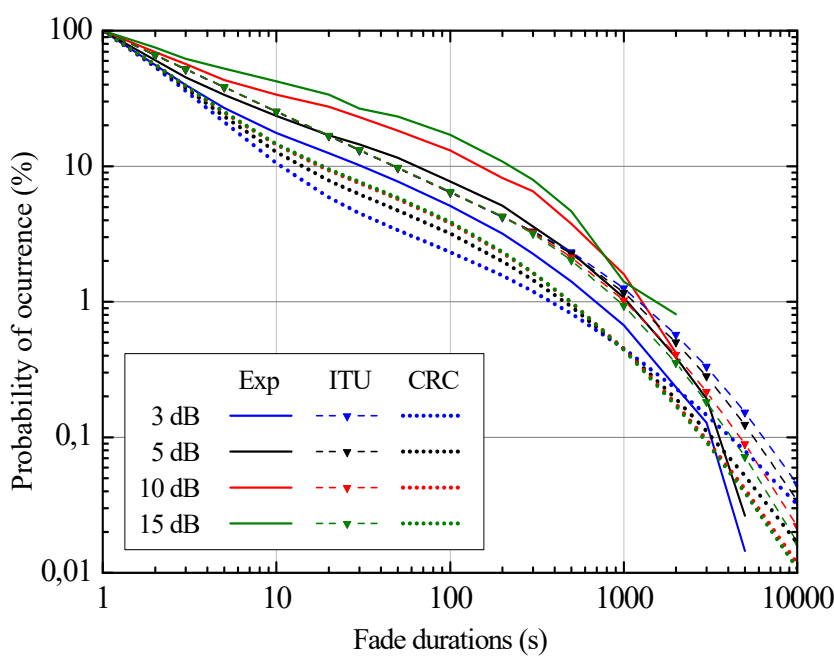

Figure 5. Probability of occurrence of fades exceeding a given duration (average year).

The CRC model under-estimates almost all the time the experimental distributions, whereas ITU predictions stay closer to the experimental distributions, particularly for 
the $5 \mathrm{~dB}$ level. However, it must be noted that two years of measurement are probably not enough to obtain the necessary statistical accuracy.

The total fading time distributions are presented in Figure 6 . The experimental and ITU distributions are very close up to a fade duration of approximately $200 \mathrm{~s}$, then the difference increases rapidly.

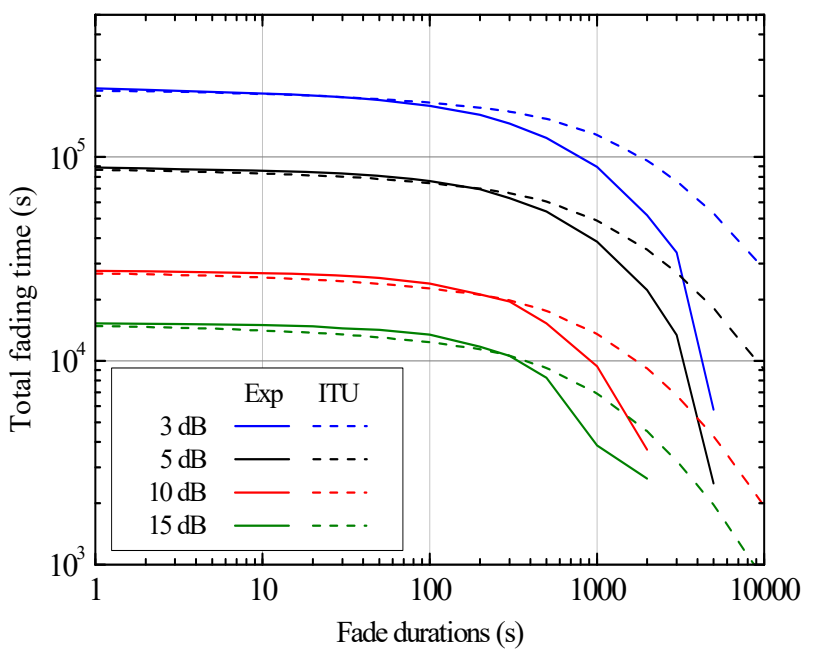

Figure 6. Total fading time (average year).

In the distributions (experimental and ITU) of total fraction of time shown in Figure 7 it can be seen that ITU distributions under-estimate the experimental fraction of time for the shorter durations up to $200 \mathrm{~s}$, where they cross the experimental curves, so that predictions overestimate the experimental values for the higher durations.

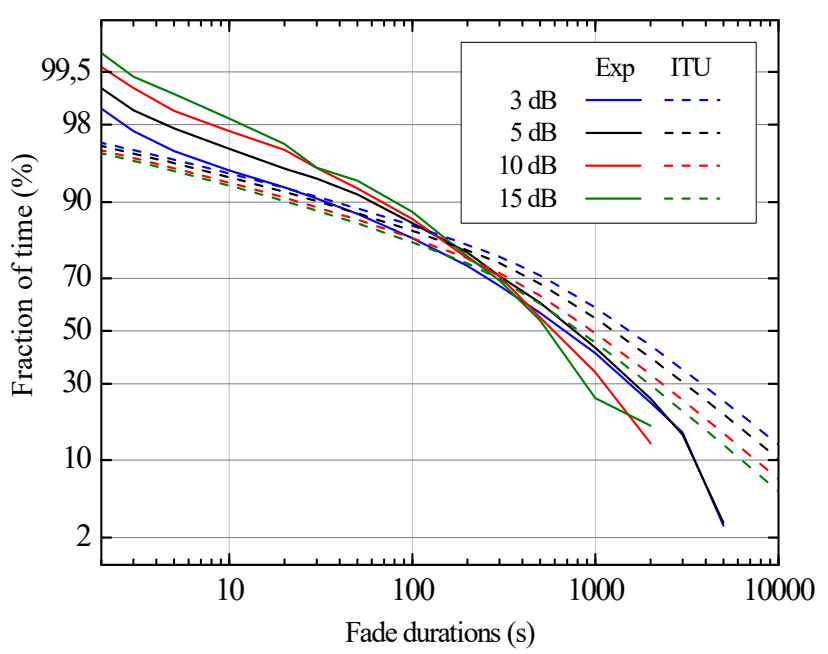

Figure 7. Total fraction of fading time (average year).

\section{Conclusions}

The first results of two years of measurements of the Alphasat Q-band beacon have been obtained in Madrid, Spain. The availability of the measurements is very high. Large variability is observed in the monthly experimental attenuation distributions, within each year and between the same months of both years. The yearly distributions are also very different. Excess and total attenuation time series have been obtained. The latter include gas attenuation, obtained using GNSS measurements.

The experimental statistics of fade dynamics have been compared with with ITU and CRC prediction models achieving results in some cases relatively close to the models. On the other hand, the variability of the statistics corresponding to different years is relevant and must be taken into account, as it is attributed to the climatic variability characteristic of Madrid. It is concluded that more measurements are needed to have more stable results.

\section{Acknowledgements}

This work was supported in part by the Ministry of Economy and Competitiveness of Spain through the TEC2014-57821-R project, and by the Regional Government of Comunidad Autónoma de Madrid, Spain under project S2013/ICE-3000.

\section{References}

1. Chia-Chin Chong, K. Hamaguchi, and P.F.M. Smulders, "Millimeter-Wave Wireless Communications Systems: Theory and Applications," EURASIP Journal on Wireless Communications and Networking, p. 2, 2007.

2. J.M. Riera, D. Pimienta-del-Valle, P. Garcia-del-Pino, G. Siles, and A. Benarroch, "Alphasat Propagation Experiment in Madrid: Results on Excess and Total Attenuation," in EuCAP 2017, Paris, France, March 2017.

3. J.M. Riera, P. Garcia-del-Pino, G. Siles, and A. Benarroch, "Propagation Experiment in Madrid using the Alphasat Q-Band beacon," in Proc. of EUCAP 2014, The Hague, The Netherlands, 6-11 April 2014, pp. 1035-39.

4. J.M. García Rubia, J.M. Riera, P. García del Pino, G.A. Siles, and A. Benarroch, "Experimental assessment of slant-path rain attenuation variability in the Ka-band," Int. J. Satell. Commun. Network, vol. 34, no. 2, pp. 155-170, March/April 2016.

5. ITU-R, "Prediction method of fade dynamics on Earthspace paths," Recommendation P. 1623-1, 2005.

6. M. Cheffena and C. Amaya, "Prediction Model of Fade Duration Statistics for Satellite Links Between 10-50 GHz," IEEE Antennas and Wireless Propagation Letters, vol. 7, pp. 260-263, 2008. 